\title{
JOINT DISTRIBUTION OF THE COVER TIME AND THE LAST VISITED POINT OF FINITE MARKOV CHAINS
}

\author{
Takuya OHWA and Tomoyuki SHIRAI
}

(Received 10 May 2007)

\begin{abstract}
We consider a Markov chain on a finite state space and obtain an expression of the joint distribution of the cover time and the last point visited by the Markov chain. As a corollary, we obtain the spectral representation of the distribution of the cover time.
\end{abstract}

\section{Introduction}

The cover time $C_{X}$ of a Markov chain on a finite set $X$ is the time taken to visit all points of $X$. The asymptotics of the expectation of the cover times for growing graphs have been investigated by many authors (cf. $[2,3]$ and the reference therein). The cover time can be represented as an absorption time in the Markov chain with states (present position and set of positions already visited by the Markov chain) as mentioned in [1]. In the present paper, we consider this extended Markov chain and obtain an expression of the joint distribution of the cover time and the last visited point by using the joint distributions of the exit time and exit point of the original Markov chain from a subset of $X$.

Let $X$ be a finite set with cardinality $|X|=N$ and $P=(p(x, y))_{x, y \in X}$ an $m$ symmetric transition probability matrix on $X$. We consider a continuous time random walk $\left(w_{t},\left\{P_{x}\right\}_{x \in X}\right)$ on $X$ with generator $\Delta=P-I$ and the cover time $C_{X}$ of $X$ defined as $C_{X}=\inf \left\{t>0 ;\left\{w_{s} ; 0 \leq s \leq t\right\}=X\right\}$ or $C_{X}=\max _{a \in X} \sigma_{a}$, where $\sigma_{a}$ is the first hitting time to $a$. Let $\mathcal{C}$ be the totality of non-empty connected subsets of $X$. Here a subset $\Lambda \subset X$ is said to be connected (with respect to $P$ ) if the random walk starting at $x$ can reach $y$ within $\Lambda$ for any $x, y \in \Lambda$; in other words, the submatrix $P_{\Lambda}=(p(x, y))_{x, y \in \Lambda}$ is irreducible. Throughout this paper, we assume that $X$ itself is connected. For $\Lambda \in \mathcal{C}$, we define a 1-neighborhood $\Lambda^{(1)}$ of $\Lambda$ by

$$
\Lambda^{(1)}=\Lambda \cup\{y \in X ; \exists x \in \Lambda \text { s.t. } p(x, y)>0\}
$$

and put $\mathcal{D}=\left\{\Lambda \in \mathcal{C} ; \Lambda \neq X, \Lambda^{(1)}=X\right\}$ and $\mathcal{D}_{x}=\{\Lambda \in \mathcal{D} ; \Lambda \ni x\}$ for $x \in X$.

THEOREM 1. Let $\left(w_{t},\left\{P_{x}\right\}_{x \in X}\right)$ be an $m$-symmetric, continuous time random walk on $X$ with generator $\Delta, C_{X}$ the cover time of $X$ by the random walk and $T_{\Lambda}$ the first exit time from

2000 Mathematics Subject Classification: Primary 60J27;

Secondary $15 \mathrm{~A} 18$.

Keywords and Phrases: Markov chain; cover time; last visited point. 
a set $\Lambda$ defined as $T_{\Lambda}=\min _{a \in \Lambda^{c}} \sigma_{a}$. Then,

$$
\frac{P_{x}\left(C_{X} \in d t ; w_{C_{X}}=y\right)}{d t}=\sum_{\Lambda \in \mathcal{D}_{x} \backslash \mathcal{D}_{y}}(-1)^{N-|\Lambda|+1} \frac{P_{x}\left(T_{\Lambda} \in d t ; w_{T_{\Lambda}}=y\right)}{d t} .
$$

In particular, any moment of $C_{X}$ and $w_{C_{X}}$ can be expressed as the alternating sum of those of $T_{\Lambda}$ :

$$
E_{x}\left[f\left(C_{X}, w_{C_{X}}\right)\right]=\sum_{\Lambda \in \mathcal{D}_{x}}(-1)^{N-|\Lambda|+1} E_{x}\left[f\left(T_{\Lambda}, w_{T_{\Lambda}}\right)\right]
$$

for any measurable function $f:[0, \infty) \times X \rightarrow \mathbb{R}$ whenever both sides make sense.

We remark that Theorem 1 holds for discrete-time Markov chains under the obvious modification. From Theorem 1, we obtain the spectral representation of the probability density of the cover time $C_{X}$.

COROLlary 1. Let $\left(w_{t},\left\{P_{x}\right\}_{x \in X}\right)$ be as in the theorem. Let $\left\{\xi_{i, \Lambda}\right\}_{i=1}^{|\Lambda|}$ be the eigenvalues of $-\Delta_{\Lambda}$ and $\left\{\varphi_{i, \Lambda}\right\}_{i=1}^{|\Lambda|}$ the corresponding orthonormal system of eigenfunctions of $\Delta_{\Lambda}=$ $(\Delta(x, y))_{x, y \in \Lambda}$ with respect to the inner product $\langle f, g\rangle_{m, \Lambda}=\sum_{x \in \Lambda} f(x) g(x) m(x)$. Then,

$$
\frac{P_{x}\left(C_{X} \in d t\right)}{d t}=\sum_{\Lambda \in \mathcal{D}_{x}}(-1)^{N-|\Lambda|+1} \sum_{i=1}^{|\Lambda|}\left\langle\mathbf{1}_{\Lambda}, \varphi_{i, \Lambda}\right\rangle_{m, \Lambda} \cdot \xi_{i, \Lambda} e^{-\xi_{i, \Lambda} t} \varphi_{i, \Lambda}(x),
$$

where $\mathbf{1}_{\Lambda}$ is the constant function on $\Lambda$ taking the value 1 .

\section{Some examples}

We consider a finite graph $G=(V(G), E(G))$ with $X=V(G)$ and the transition matrix $P$ corresponding to the simple random walk on $G$, i.e. $p(x, y)=1 / \operatorname{deg}(x)$ where $y$ is any neighbor point and $\operatorname{deg}(x)$ is the degree of $x$.

Example 1. Let $C_{N}$ be a cycle of length $N$. We denote the vertex set by $X=\{0,1, \ldots$, $N-1\}$ and

$$
p(x, y)= \begin{cases}1 / 2, & y=x-1, x+1 \quad \bmod N \\ 0, & \text { otherwise. }\end{cases}
$$

Then, $\mathcal{D}_{0}=\{X \backslash\{k\} ; k=1, \ldots, N-1\} \cup\{X \backslash\{k, k+1\} ; k=1, \ldots, N-2\}$. It is easy to see that $E_{0}\left[C_{X}\right]=N(N-1) / 2$. Moreover, the Laplace transform of $C_{X}$ is given by

$$
\begin{aligned}
E_{0}\left[e^{-\lambda C_{X}}\right] & =\sum_{\Lambda \in \mathcal{D}_{0}}(-1)^{N-|\Lambda|+1} E_{0}\left[e^{-\lambda T_{\Lambda}}\right] \\
& =\sum_{k=1}^{N-1} \frac{\mu_{+}^{k}-\mu_{-}^{k}+\mu_{+}^{N-k}-\mu_{-}^{N-k}}{\mu_{+}^{N}-\mu_{-}^{N}}-\sum_{k=1}^{N-2} \frac{\mu_{+}^{k}-\mu_{-}^{k}+\mu_{+}^{N-1-k}-\mu_{-}^{N-1-k}}{\mu_{+}^{N-1}-\mu_{-}^{N-1}} \\
& =\frac{2\left(\mu_{+}^{1 / 2}+\mu_{-}^{1 / 2}\right)}{\left(\mu_{+}^{N / 2}+\mu_{-}^{N / 2}\right)\left(\mu_{+}^{(N-1) / 2}+\mu_{-}^{(N-1) / 2}\right)},
\end{aligned}
$$

where $\mu_{ \pm}=\mu_{ \pm}(\lambda)=e^{\lambda} \pm \sqrt{e^{2 \lambda}-1}$. Since $\mu_{ \pm}\left(\lambda / N^{2}\right)^{N} \rightarrow e^{ \pm \sqrt{2 \lambda}}$ as $N \rightarrow \infty$, we obtain

$$
\lim _{N \rightarrow \infty} E_{0}\left[e^{-\lambda C_{X} / N^{2}}\right]=\left(\cosh \sqrt{\frac{\lambda}{2}}\right)^{-2} \text {. }
$$


The right-hand side is the Laplace transform of the distribution of the cover time $C$ for the standard Brownian motion on $S^{1}$ :

$$
\frac{P(C \in d t)}{d t}=\frac{4}{\sqrt{2 \pi t^{3}}} \sum_{m=1}^{\infty}(-1)^{m-1} m^{2} e^{-m^{2} / 2 t},
$$

which was first obtained by Feller [4].

We note that

$$
\mathcal{D}_{0} \backslash \mathcal{D}_{y}= \begin{cases}\left\{\{y\}^{c},\{y-1, y\}^{c},\{y, y+1\}^{c}\right\}, & y \neq 1, N-1, \\ \left\{\{1\}^{c},\{1,2\}^{c}\right\}, & y=1, \\ \left\{\{N-1\}^{c},\{N-2, N-1\}^{c}\right\}, & y=N-1,\end{cases}
$$

and $\quad P_{0}\left(w_{T_{\{y, y+1\}^{c}}}=y\right)=(N-y-1) /(N-1), \quad P_{0}\left(w_{T_{\{y-1, y\}}}=y\right)=(y-1) /(N-1)$. Then, we obtain

$$
\begin{aligned}
P_{0}\left(w_{C_{X}}=y\right) & =\sum_{\Lambda \in \mathcal{D}_{0} \backslash \mathcal{D}_{y}}(-1)^{N-|\Lambda|+1} P_{0}\left(w_{T_{\Lambda}}=y\right) \\
& =\frac{1}{N-1}
\end{aligned}
$$

for any $y \in\{1,2, \ldots, N-1\}$, which is a well-known fact for the last vertex visited by a random walk (cf. [5]).

Example 2. Let $G_{j}(j=1, \ldots, m)$ be path graphs of length $N$ and $G$ the star-like graph obtained from $G_{j}$ by identifying their one end point with a vertex, say 0 . The vertex set of $G$ is then identified with the set $X=\{(k, j) ; k=1,2, \ldots, N, j=1, \ldots, m\} \cup\{0\}$. In this case,

$$
\mathcal{D}_{0}=\left\{X \backslash \bigcup_{j \in I}\{(N, j)\} ; I \subset\{1,2, \ldots, m\}, I \neq \emptyset\right\} .
$$

Some computation shows that

$$
\begin{aligned}
E_{0}\left[e^{-\lambda C_{X}}\right] & =\sum_{\Lambda \in \mathcal{D}_{0}}(-1)^{N-|\Lambda|+1} E_{0}\left[e^{-\lambda T_{\Lambda}}\right] \\
& =\sum_{k=1}^{m} \sum_{\substack{I \subset\{1,2, \ldots, m\} \\
|I|=k}}(-1)^{k-1} \frac{2 k\left(\mu_{+}^{N-1}+\mu_{-}^{N-1}\right)}{m \mu_{+}^{2 N-1}+m \mu_{-}^{2 N-1}+\left(\mu_{+}+\mu_{-}\right)(2 k-m)} \\
& =\sum_{k=1}^{m}(-1)^{k-1}\left(\begin{array}{l}
m \\
k
\end{array}\right) \frac{2 k\left(\mu_{+}^{N-1}+\mu_{-}^{N-1}\right)}{m \mu_{+}^{2 N-1}+m \mu_{-}^{2 N-1}+\left(\mu_{+}+\mu_{-}\right)(2 k-m)} \\
& =\prod_{j=1}^{m} \frac{2 j\left(\mu_{+}^{N-1}+\mu_{-}^{N-1}\right)}{m \mu_{+}^{2 N-1}+m \mu_{-}^{2 N-1}+\left(\mu_{+}+\mu_{-}\right)(2 j-m)}
\end{aligned}
$$

where $\mu_{ \pm}$is the same as in the previous example and we used the identity

$$
\sum_{k=1}^{m}(-1)^{k-1}\left(\begin{array}{l}
m \\
k
\end{array}\right) \frac{k}{k+x}=\prod_{j=1}^{m} \frac{j}{j+x}
$$


for the last equality. Hence, we see that

$$
\begin{aligned}
\lim _{N \rightarrow \infty} E_{0}\left[e^{-\lambda C_{X} / N^{2}}\right] & =\frac{1}{\cosh \sqrt{2 \lambda}} \prod_{j=1}^{m-1}\left(\frac{j}{j+m \sinh ^{2} \sqrt{2 \lambda}}\right) \\
& =E_{0}\left[e^{-\lambda T}\right] \prod_{j=1}^{m-1} E_{0}\left[e^{-\lambda T_{j}}\right],
\end{aligned}
$$

where $T$ is the absorption time at 1 for the standard Brownian motion on $(0,1)$ with reflecting boundary at 0 and $T_{j}$ is the absorption time at 2 for the skew Brownian motion on $(0,2)$ with skew parameter $j / m$ at 1 and reflecting boundary at 0 . We refer to [6] for the skew Brownian motion.

\section{Proof of the theorem}

Throughout this paper, we assume that $X$ is connected. Let $\mathcal{C}_{k}$ be the totality of connected subsets of $X$ with cardinality $k$ and $\mathcal{C}=\bigcup_{k=1}^{N} \mathcal{C}_{k}$. We define an extension $Y$ of $X$ by

$$
Y=\left\{(x, A) \in X \times 2^{X} ; A \in \mathcal{C}, x \in A\right\}
$$

and a transition probability $Q=(q(\eta, \xi))_{\eta, \xi \in Y}$ on $Y$ by

$$
q((x, A),(y, B))= \begin{cases}p(x, y), & \text { if } B=A \cup\{y\}, \\ 0, & \text { otherwise }\end{cases}
$$

Let $\left(w_{t}, A_{t}\right)$ be a continuous-time random walk on $Y$ with generator $Q-I$. Then it is obvious that the first marginal process is equal in law to the original random walk on $X$ and the cover time is equal to the first hitting time for $A_{t}$ to $X$.

We decompose $Y$ into the level sets as follows:

$$
Y=\bigcup_{k=1}^{N} L_{k}=\bigcup_{k=1}^{N} \bigcup_{\Lambda \in \mathcal{C}_{k}} L_{\Lambda},
$$

where $L_{\Lambda}=\{(x, \Lambda) \in Y ; x \in \Lambda\}$ for $\Lambda \in \mathcal{C}$ and $L_{k}=\bigcup_{\Lambda \in \mathcal{C}_{k}} L_{\Lambda}$ for $1 \leq k \leq N$. Since $\left|L_{\Lambda}\right|=|\Lambda|$, one can see that $|Y|=\sum_{k=1}^{N} k\left|\mathcal{C}_{k}\right|$.

For given two subsets $\Lambda, \Lambda^{\prime} \in \mathcal{C}$, a submatrix $Q_{\Lambda \Lambda^{\prime}}$ of $Q$ is defined by $Q_{\Lambda \Lambda^{\prime}}=$ $(q(\eta, \xi))_{\eta \in L_{\Lambda}, \xi \in L_{\Lambda^{\prime}}}$ and, for simplicity, denote $Q_{\Lambda \Lambda}$ by $Q_{\Lambda}$. Moreover, put $Q_{i j}=$ $(q(\eta, \xi))_{\eta \in L_{i}, \xi \in L_{j}}$ and $Q_{i}=Q_{i i}$. The matrix $Q$ is an upper triangular and tridiagonal block matrix.

LEMMA 1. We have:

(i) $Q_{i j}=O$ unless $j=i, i+1$ and $Q_{N}=P$;

(ii) $Q_{k}=\bigoplus_{\Lambda \in \mathcal{C}_{k}} Q_{\Lambda}$, i.e. $Q_{\Lambda \Lambda^{\prime}}=O$ if $\Lambda, \Lambda^{\prime} \in \mathcal{C}_{k}$ and $\Lambda \neq \Lambda^{\prime}$; furthermore, $Q_{\Lambda}=$ $P_{\Lambda}=(p(x, y))_{x, y \in \Lambda}$.

Proof. It is easy to see from the definitions of $Y$ and $Q$. 
Recall that $P$ is said to be $m$-symmetric or symmetrizable with respect to $m$ if there exists a positive function $m: X \rightarrow(0, \infty)$ such that $m(x) p(x, y)=m(y) p(y, x)$ for every $x, y \in X$. Even if $P$ is symmetrizable, the matrix $Q$ itself can never be symmetrizable from Lemma 1(i) and the fact $Q_{i, i+1} \neq O$ for $i=1,2, \ldots, N-1$; however, each restriction on diagonal blocks is always symmetrizable.

Lemma 2. Suppose that $P$ is $m$-symmetric. Define a function $m_{k}: L_{k} \rightarrow(0, \infty)$ by

$$
m_{k}(x, A)=m(x), \quad(x, A) \in L_{k} .
$$

Then, $Q_{k}$ is $m_{k}$-symmetric for every $1 \leq k \leq N$. In particular, all eigenvalues of $Q$ are real and

$$
\operatorname{Spec}(Q)=\bigcup_{k=1}^{N} \bigcup_{\Lambda \in \mathcal{C}_{k}} \operatorname{Spec}\left(P_{\Lambda}\right),
$$

where $P_{\Lambda}=(p(x, y))_{x, y \in \Lambda}$.

Proof. For $x, y \in A$, we see that

$$
\begin{aligned}
& m_{k}((x, A)) q((x, A),(y, A)) \\
& \quad=m(x) p(x, y)=m(y) p(y, x)=m_{k}((y, A)) q((y, A),(x, A)) .
\end{aligned}
$$

Since the matrix $Q$ is an upper triangular block matrix from Lemma 1(i), the set of eigenvalues are the union of those of diagonal blocks $Q_{k}, 1 \leq k \leq N$; moreover, the union of those of $Q_{\Lambda}, \Lambda \in \mathcal{C}$ from Lemma 1(ii). The assertion follows from the fact that each $Q_{\Lambda}$ has the same real eigenvalues as $P_{\Lambda}$.

Since $P_{\Lambda}$ is $\left.m\right|_{\Lambda}$-symmetric, one can find an orthonormal system of eigenfunctions $\left\{\varphi_{i, \Lambda}\right\}_{i=1, \ldots,|\Lambda|}$ of $\ell^{2}\left(\Lambda,\left.m\right|_{\Lambda}\right)$ for each $\Lambda \in \mathcal{C}$ such that $P_{\Lambda} \varphi_{i, \Lambda}=\lambda_{i, \Lambda} \varphi_{i, \Lambda}$. It is easy to check that $\psi_{i, \Lambda}:=m \varphi_{i, \Lambda}$ is the eigenfunction corresponding to $\lambda_{i, \Lambda}$ of the transposed matrix $P_{\Lambda}^{\mathrm{T}}$ of $P_{\Lambda}$. All of the eigenfunctions of $Q$ and $Q^{\mathrm{T}}$ are obtained from $\left\{\varphi_{i, \Lambda}, i=1, \ldots,|\Lambda|, \Lambda \in \mathcal{C}\right\}$ and $\left\{\psi_{i, \Lambda}, i=1, \ldots,|\Lambda|, \Lambda \in \mathcal{C}\right\}$ by their extensions to $Y$ (see Lemma 6). In what follows, we keep the notation $\lambda_{i, \Lambda}, \varphi_{i, \Lambda}$ and $\psi_{i, \Lambda}$ for the eigenvalues and eigenfunctions of $P_{\Lambda}$ and $P_{\Lambda}^{\mathrm{T}}$, and we often regard matrices $P_{\Lambda}, P, Q$, etc., as operators on suitable function spaces.

Lemma 3. Let $\Lambda, \Lambda^{\prime} \in \mathcal{C}$ and $\Lambda^{(1)}$ be the 1-neighborhood of $\Lambda$. Then,

$$
\sum_{\substack{A \in \mathcal{C} \\ \Lambda \subset A \subset \Lambda^{(1)} \cap \Lambda^{\prime}}}(-1)^{|A|-|\Lambda|}=\delta_{\Lambda, \Lambda^{\prime}} .
$$

Proof. It suffices to show the case where $\Lambda \subsetneq \Lambda^{\prime}$, otherwise the assertion is trivial. Put $\partial \Lambda=\Lambda^{(1)} \backslash \Lambda$ and $n=\left|\partial \Lambda \cap \Lambda^{\prime}\right|(\geq 1)$. Since $\Lambda \cup B \in \mathcal{C}$ for any $B \subset \partial \Lambda \cap \Lambda^{\prime}$, we have

$$
\begin{aligned}
\sum_{\substack{A \in \mathcal{C} \\
\Lambda \subset A \subset \Lambda^{(1)} \cap \Lambda^{\prime}}}(-1)^{|A|-|\Lambda|} & =\sum_{k=0}^{n} \sum_{\substack{B \subset \partial \Lambda \cap \Lambda^{\prime} \\
|B|=k}}(-1)^{|\Lambda \cup B|-|\Lambda|} \\
& =\sum_{k=0}^{n}\left(\begin{array}{l}
n \\
k
\end{array}\right)(-1)^{k}=0 .
\end{aligned}
$$


Let $\ell^{2}(Y)$ and $\ell^{2}(\Lambda)$ be inner product spaces of real functions on $Y$ and $\Lambda$, respectively, with inner products

$$
(F, G)_{\ell^{2}(Y)}=\sum_{(x, A) \in Y} F(x, A) G(x, A), \quad(f, g)_{\ell^{2}(\Lambda)}=\sum_{x \in \Lambda} f(x) g(x) .
$$

We denote by $L^{*}$ the dual operator of $L: \ell^{2}(\Lambda) \rightarrow \ell^{2}(Y)$ defined by the formula $(L f, F)_{\ell^{2}(Y)}=\left(f, L^{*} F\right)_{\ell^{2}(\Lambda)}$ for any $f \in \ell^{2}(\Lambda)$ and $F \in \ell^{2}(Y)$.

Lemma 4. Define two operators $U_{\Lambda}, D_{\Lambda}: \ell^{2}(\Lambda) \rightarrow \ell^{2}(Y)$ by

$$
\begin{aligned}
& \left(U_{\Lambda} f\right)(x, A)= \begin{cases}f(x), & A \subset \Lambda, \\
0, & \text { otherwise, }\end{cases} \\
& \left(D_{\Lambda} f\right)(x, A)= \begin{cases}(-1)^{|A|-|\Lambda|} f(x) I_{\Lambda}(x), & \Lambda \subset A \subset \Lambda^{(1)}, \\
0, & \text { otherwise, }\end{cases}
\end{aligned}
$$

where $I_{\Lambda} \in \ell^{2}(X)$ is the indicator function of $\Lambda$. Then, the dual operators $U_{\Lambda}^{*}, D_{\Lambda}^{*}: \ell^{2}(Y) \rightarrow$ $\ell^{2}(\Lambda)$ are given by

$$
\begin{aligned}
& \left(U_{\Lambda}^{*} F\right)(x)=\sum_{\substack{A \in \mathcal{C} \\
A \subset \Lambda, A \ni x}} F(x, A), \\
& \left(D_{\Lambda}^{*} F\right)(x)=\sum_{\substack{A \in \mathcal{C} \\
\Lambda \subset A \subset \Lambda^{(1)}, A \ni x}}(-1)^{|A|-|\Lambda|} F(x, A) .
\end{aligned}
$$

Moreover,

$$
\left(U_{\Lambda} f, D_{\Lambda^{\prime}} g\right)_{\ell^{2}(Y)}= \begin{cases}(f, g)_{\ell^{2}(\Lambda)}, & \Lambda=\Lambda^{\prime}, \\ 0, & \Lambda \neq \Lambda^{\prime},\end{cases}
$$

for any $f \in \ell^{2}(\Lambda)$ and $g \in \ell^{2}\left(\Lambda^{\prime}\right)$.

Proof. The expression of the dual operators are easily obtained by a direct computation. For $f \in \ell^{2}(\Lambda)$ and $g \in \ell^{2}\left(\Lambda^{\prime}\right)$, we see that

$$
\begin{aligned}
\left(U_{\Lambda} f, D_{\Lambda^{\prime}} g\right)_{\ell^{2}(Y)} & =\sum_{\substack{(x, A) \in Y \\
A \subset \Lambda \text { and } \Lambda^{\prime} \subset A \subset\left(\Lambda^{\prime}\right)^{(1)}}} f(x)(-1)^{|A|-\left|\Lambda^{\prime}\right|} g(x) I_{\Lambda^{\prime}}(x) \\
= & \sum_{\substack{(x, A) \in Y \\
x \in \Lambda^{\prime} \text { and } \Lambda^{\prime} \subset A \subset\left(\Lambda^{\prime}\right)^{(1)} \cap \Lambda}} f(x) g(x)(-1)^{|A|-\left|\Lambda^{\prime}\right|} \\
= & \sum_{x \in \Lambda^{\prime}} f(x) g(x) \sum_{\substack{A \in \mathcal{C} \\
\Lambda^{\prime} \subset A \subset\left(\Lambda^{\prime}\right)^{(1)} \cap \Lambda}}(-1)^{|A|-\left|\Lambda^{\prime}\right|} \\
= & (f, g)_{\ell^{2}\left(\Lambda^{\prime}\right)} \cdot \delta_{\Lambda^{\prime}, \Lambda} .
\end{aligned}
$$

Here the last equality follows from Lemma 3.

Lemma 5. For any $\Lambda \in \mathcal{C}$, two operators $U_{\Lambda}$ and $D_{\Lambda}$ are intertwiners in the sense that

$$
Q U_{\Lambda}=U_{\Lambda} P_{\Lambda}, \quad Q^{\mathrm{T}} D_{\Lambda}=D_{\Lambda} P_{\Lambda}^{\mathrm{T}} .
$$


Proof. Set

$$
\chi_{\Lambda}(B)= \begin{cases}1, & B \subset \Lambda \\ 0, & \text { otherwise }\end{cases}
$$

Noting that $U_{\Lambda} f(x, A)=f(x) \chi_{\Lambda}(A)$ on $Y$, we have

$$
\begin{aligned}
Q U_{\Lambda} f(x, A) & =\sum_{(y, B) \in Y} Q((x, A),(y, B)) U_{\Lambda} f(y, B) \\
& =\sum_{y \in X} p(x, y) f(y) \chi_{\Lambda}(A \cup\{y\}) \\
& =\sum_{y \in \Lambda} p(x, y) f(y) \chi_{\Lambda}(A) \\
& =P_{\Lambda} f(x) \chi_{\Lambda}(A)=U_{\Lambda} P_{\Lambda} f(x, A)
\end{aligned}
$$

for $f \in \ell^{2}(\Lambda)$. Since the set $\left\{(y, B) \in Y ; y \in \Lambda, \Lambda \subset B \subset \Lambda^{(1)}, A=B \cup\{x\}\right\}$ is equal to the product set $\Lambda \times\left\{B \in \mathcal{C} ; \Lambda \subset B \subset \Lambda^{(1)}, A=B \cup\{x\}\right\}$, we see that

$$
\begin{aligned}
Q^{\mathrm{T}} D_{\Lambda} f(x, A) & =\sum_{(y, B) \in Y} Q^{\mathrm{T}}((x, A),(y, B)) D_{\Lambda} f(y, B) \\
& =\sum_{\substack{(y, B) \in Y \\
y \in \Lambda \text { and } A=B \cup\{x\}}} p(y, x)(-1)^{|B|-|\Lambda|} f(y) \zeta_{\Lambda}(B) \\
& =\sum_{y \in \Lambda} p(y, x) f(y) \times \sum_{\substack{B \in \mathcal{C} \\
A=B \cup\{x\}}}(-1)^{|B|-|\Lambda|} \zeta_{\Lambda}(B),
\end{aligned}
$$

where

$$
\zeta_{\Lambda}(B)= \begin{cases}1, & \Lambda \subset B \subset \Lambda^{(1)} \\ 0, & \text { otherwise. }\end{cases}
$$

We consider three cases: (i) $x \notin \Lambda^{(1)}$, (ii) $x \in \Lambda$ and (iii) $x \in \Lambda^{(1)} \backslash \Lambda$. If $x \notin \Lambda^{(1)}$, then it is clear that $\sum_{y \in \Lambda} p(y, x) f(y)=0$ by the definition of $\Lambda^{(1)}$. We note that $A=B \cup\{x\}$ implies $B=A$ or $B=A \backslash\{x\}$. If $x \in \Lambda$ and $\Lambda \subset B \subset \Lambda^{(1)}$, then $B=A$ and, hence,

$$
Q^{\mathrm{T}} D_{\Lambda} f(x, A)=\sum_{y \in \Lambda} p(y, x) f(y) \times(-1)^{|A|-|\Lambda|} \zeta_{\Lambda}(A)=D_{\Lambda}\left(P_{\Lambda}^{\mathrm{T}} f\right)(x, A) .
$$

If $x \in \Lambda^{(1)} \backslash \Lambda$ and $\Lambda \subset B \subset \Lambda^{(1)}$, then $\Lambda \subset A \subset \Lambda^{(1)}$. Since $\Lambda \subset A \subset \Lambda^{(1)}$ and $x \in \Lambda^{(1)} \backslash \Lambda$ imply that $A \backslash\{x\} \in \mathcal{C}$ and $\Lambda \subset A \backslash\{x\} \subset \Lambda^{(1)}$, we see that

$$
\sum_{\substack{B \in \mathcal{C} \\ A=B \cup\{x\}}}(-1)^{|B|-|\Lambda|} \zeta_{\Lambda}(B)=(-1)^{|A|-|\Lambda|}+(-1)^{|A \backslash\{x\}|-|\Lambda|}=0 .
$$

On the other hand, $D_{\Lambda} P_{\Lambda}^{\mathrm{T}} f(x, A)=0$ unless $x \in \Lambda$. Therefore, we conclude that $Q^{\mathrm{T}} D_{\Lambda} f(x, A)=D_{\Lambda} P_{\Lambda}^{\mathrm{T}} f(x, A)$.

Lemma 6. The systems $\left\{U_{\Lambda} \varphi_{i, \Lambda}, \Lambda \in \mathcal{C}, i=1, \ldots,|\Lambda|\right\}$ and $\left\{D_{\Lambda} \psi_{i, \Lambda}, \Lambda \in \mathcal{C}, i=1\right.$, $\ldots,|\Lambda|\}$ are the eigenfunctions corresponding to the eigenvalues $\left\{\lambda_{i, \Lambda}, \Lambda \in \mathcal{C}, i=\right.$ $1, \ldots,|\Lambda|\}$ of $Q$ and $Q^{\mathrm{T}}$, respectively; each system forms a basis of $\ell^{2}(Y)$. Moreover, they 
are bi-orthogonal in the sense that

$$
\left(U_{\Lambda} \varphi_{i, \Lambda}, D_{\Lambda^{\prime}} \psi_{j, \Lambda^{\prime}}\right)_{\ell^{2}(Y)}=\delta_{i, j} \delta_{\Lambda, \Lambda^{\prime}}
$$

Proof. This follows immediately from Lemmas 4 and 5.

We obtain a spectral representation for the transition kernel of the non-symmetrizable transition operator $Q$.

PROPOSITION 1. The kernel of the transition operator $Q$ has the following spectral representation:

$$
Q(\eta, \xi)=\sum_{\Lambda \in \mathcal{C}} \sum_{i=1}^{|\Lambda|} \lambda_{i, \Lambda}\left(U_{\Lambda} \varphi_{i, \Lambda}\right)(\eta)\left(D_{\Lambda} \psi_{i, \Lambda}\right)(\xi) .
$$

Moreover, if $\eta=(x, A)$ and $\xi=(y, B)$, then

$$
Q^{n}((x, A),(y, B))=\sum_{\substack{\Lambda \in \mathcal{C} \\ x, y \in \Lambda, A \subset \Lambda \subset B \subset \Lambda^{(1)}}}(-1)^{|B|-|\Lambda|} P_{\Lambda}^{n}(x, y)
$$

for $n=0,1,2 \ldots$

Proof. Note that $\delta_{\eta}=\sum_{\Lambda \in \mathcal{C}} \sum_{i=1}^{|\Lambda|}\left(U_{\Lambda} \varphi_{i, \Lambda}, \delta_{\eta}\right)_{\ell^{2}(Y)} D_{\Lambda} \psi_{i, \Lambda}$ by Lemmas 4 and 6 . Then, we obtain

$$
\begin{aligned}
Q(\eta, \xi) & =\left(Q \delta_{\xi}, \delta_{\eta}\right)_{\ell^{2}(Y)}=\sum_{\Lambda \in \mathcal{C}} \sum_{i=1}^{|\Lambda|}\left(U_{\Lambda} \varphi_{i, \Lambda}, \delta_{\eta}\right)_{\ell^{2}(Y)} \cdot\left(Q \delta_{\xi}, D_{\Lambda} \psi_{i, \Lambda}\right)_{\ell^{2}(Y)} \\
& =\sum_{\Lambda \in \mathcal{C}} \sum_{i=1}^{|\Lambda|}\left(U_{\Lambda} \varphi_{i, \Lambda}, \delta_{\eta}\right)_{\ell^{2}(Y)} \cdot\left(\delta_{\xi}, Q^{\mathrm{T}} D_{\Lambda} \psi_{i, \Lambda}\right)_{\ell^{2}(Y)} \\
& =\sum_{\Lambda \in \mathcal{C}} \sum_{i=1}^{|\Lambda|} \lambda_{i, \Lambda}\left(U_{\Lambda} \varphi_{i, \Lambda}\right)(\eta)\left(D_{\Lambda} \psi_{i, \Lambda}\right)(\xi) .
\end{aligned}
$$

Set $\eta=(x, A)$ and $\xi=(y, B)$. Similarly, since $Q^{n} U_{\Lambda}=U_{\Lambda} P_{\Lambda}^{n}$, we see that

$$
\begin{aligned}
Q^{n}(\eta, \xi)= & \sum_{\Lambda \in \mathcal{C}} \sum_{i=1}^{|\Lambda|}\left(U_{\Lambda} P_{\Lambda}^{n} \varphi_{i, \Lambda}\right)(\eta)\left(D_{\Lambda} \psi_{i, \Lambda}\right)(\xi) \\
= & \sum_{\substack{\Lambda \in \mathcal{C} \\
A \subset \Lambda \subset B \subset \Lambda^{(1)}}} \sum_{i=1}^{|\Lambda|}\left(P_{\Lambda}^{n} \varphi_{i, \Lambda}\right)(x) I_{\Lambda}(x)(-1)^{|B|-|\Lambda|} \psi_{i, \Lambda}(y) I_{\Lambda}(y) \\
= & \sum_{\substack{\Lambda \in \mathcal{C} \\
x, y \in \Lambda, A \subset \Lambda \subset B \subset \Lambda^{(1)}}}(-1)^{|B|-|\Lambda|} P_{\Lambda}^{n}(x, y) .
\end{aligned}
$$

We used the equality $\sum_{i=1}^{|\Lambda|} \varphi_{i, \Lambda}(y) \psi_{i, \Lambda}(y)=1$ for the last line.

Let $\ell^{2}(Y ; \Lambda)$ be the subspace of $\ell^{2}(Y)$ of functions supported by $\Lambda$. We decompose $\ell^{2}(Y)$ into the direct sum of $\ell^{2}\left(Y ; Y \backslash L_{N}\right) \oplus \ell^{2}\left(Y ; L_{N}\right)$. We define $\Pi_{1}$ and $\Pi_{2}$ to be 
the projection operators onto subspaces $\ell^{2}\left(Y ; Y \backslash L_{N}\right)$ and $\ell^{2}\left(Y ; L_{N}\right)$, respectively. We identify an element of $\ell^{2}(X)$ with that of $\ell^{2}\left(Y ; L_{N}\right)$ by the mapping $\iota: \ell^{2}(X) \rightarrow \ell^{2}\left(Y ; L_{N}\right)$ defined by

$$
\text { (ıf })(x, A)= \begin{cases}f(x), & A=X, \\ 0, & A \neq X,\end{cases}
$$

for $f \in \ell^{2}(X)$. Also we define $\Pi_{\Lambda}: \ell^{2}(X) \rightarrow \ell^{2}(\Lambda)$ by $\Pi_{\Lambda} h(x)=h(x)$ for each $x \in \Lambda$.

Lemma 7. Let $h \in \ell^{2}(X)$. Then,

$$
\begin{gathered}
\Pi_{1} U_{X} h=\sum_{\Lambda \in \mathcal{D}}(-1)^{N-|\Lambda|+1} \sum_{j=1}^{|\Lambda|}\left\langle\Pi_{\Lambda} h, \varphi_{j, \Lambda}\right\rangle_{m, \Lambda} U_{\Lambda} \varphi_{j, \Lambda}, \\
\Pi_{2} U_{X} h=\iota h,
\end{gathered}
$$

where $\mathcal{D}=\left\{\Lambda \in \mathcal{C} ; \Lambda \neq X, \Lambda^{(1)}=X\right\}$.

Proof. The second equality is trivial. First we remark that the system $\left\{U_{\Lambda} \varphi_{j, \Lambda}, \Lambda \in \mathcal{C}\right.$, $\Lambda \neq X, j=1, \ldots,|\Lambda|\}$ is a basis of $\ell^{2}\left(Y ; Y \backslash L_{N}\right)$. By Lemma 4, we have

$$
\Pi_{1} U_{X} h=\sum_{\Lambda \in \mathcal{C}} \sum_{j=1}^{|\Lambda|}\left(\Pi_{1} U_{X} h, D_{\Lambda} \psi_{j, \Lambda}\right)_{\ell^{2}(Y)} U_{\Lambda} \varphi_{j, \Lambda}
$$

for $h \in \ell^{2}(X)$. When $\Lambda=X$, it is obvious that $\left(\Pi_{1} U_{X} h, D_{X} \psi_{j, X}\right)_{\ell^{2}(Y)}=0$ since $\Pi_{1} U_{X} h \in$ $\ell^{2}\left(Y ; Y \backslash L_{N}\right)$ and $D_{X} \psi_{j, X} \in \ell^{2}\left(Y ; L_{N}\right)$. When $\Lambda \neq X$,

$$
\begin{aligned}
\left(\Pi_{1} U_{X} h, D_{\Lambda} \psi_{j, \Lambda}\right)_{\ell^{2}(Y)} & =\left(U_{X} h-\iota h, D_{\Lambda} \psi_{j, \Lambda}\right)_{\ell^{2}(Y)} \\
& =-\left(\iota h, D_{\Lambda} \psi_{j, \Lambda}\right)_{\ell^{2}(Y)} \\
& = \begin{cases}(-1)^{N-|\Lambda|+1}\left\langle\Pi_{\Lambda} h, \varphi_{j, \Lambda}\right\rangle_{m, \Lambda}, & \Lambda^{(1)}=X, \\
0, & \Lambda^{(1)} \neq X .\end{cases}
\end{aligned}
$$

Here we used the bi-orthogonality in Lemma 4 for the second equality and the definition of $D_{\Lambda}$ and $\psi_{j, \Lambda}=m \varphi_{j, \Lambda}$ for the last equality.

We define two operators $R: \ell^{2}(Y) \rightarrow \ell^{2}\left(Y ; Y \backslash L_{N}\right)$ and $S: \ell^{2}(Y) \rightarrow \ell^{2}\left(Y ; Y \backslash L_{N}\right)$ by

$$
R=\Pi_{1} Q \Pi_{1}, \quad S=\Pi_{1} Q \Pi_{2} .
$$

We remark that the operator $R$ can be regarded as an operator from $\ell^{2}\left(Y ; Y \backslash L_{N}\right)$ to itself and the system of eigenfunctions of $R,\left\{U_{\Lambda} \varphi_{i, \Lambda}, \Lambda \in \mathcal{C}, \Lambda \neq X, i=1, \ldots,|\Lambda|\right\}$, forms a basis of $\ell^{2}\left(Y ; Y \backslash L_{N}\right)$.

LEMMA 8. For $h \in \ell^{2}(X)$, we have

$$
S U_{X} h=\sum_{\Lambda \in \mathcal{D}}(-1)^{N-|\Lambda|+1} \sum_{j=1}^{|\Lambda|}\left\langle P_{\Lambda} \Lambda^{c} h, \varphi_{j, \Lambda}\right\rangle_{m, \Lambda} U_{\Lambda} \varphi_{j, \Lambda},
$$

where $\mathcal{D}=\left\{\Lambda \in \mathcal{C} ; \Lambda \neq X, \Lambda^{(1)}=X\right\}$ and $P_{\Lambda} \Lambda^{c}=\Pi_{\Lambda} P-P_{\Lambda} \Pi_{\Lambda}: \ell^{2}(X) \rightarrow \ell^{2}(\Lambda)$. 
Proof. We note that $\Pi_{1} Q=R+S$ by definition and $Q U_{X}=U_{X} P$ by Lemma 5. Since $\varphi_{j, \Lambda}$ and $U_{\Lambda} \varphi_{j, \Lambda}$ are eigenfunctions corresponding to $\lambda_{j, \Lambda}$ of $P_{\Lambda}$ and $R$, respectively, by using Lemma 7, we have

$$
\begin{aligned}
S U_{X} h & =\left(\Pi_{1} Q-R\right) U_{X} h=\Pi_{1} U_{X} P h-R \Pi_{1} U_{X} h \\
& =\sum_{\Lambda \in \mathcal{D}}(-1)^{N-|\Lambda|+1} \sum_{j=1}^{|\Lambda|}\left(\left\langle\Pi_{\Lambda} P h, \varphi_{j, \Lambda}\right\rangle_{m, \Lambda} U_{\Lambda} \varphi_{j, \Lambda}-\left\langle\Pi_{\Lambda} h, \varphi_{j, \Lambda}\right\rangle_{m, \Lambda} R U_{\Lambda} \varphi_{j, \Lambda}\right) \\
& =\sum_{\Lambda \in \mathcal{D}}(-1)^{N-|\Lambda|+1} \sum_{j=1}^{|\Lambda|}\left\langle\left(\Pi_{\Lambda} P-P_{\Lambda} \Pi_{\Lambda}\right) h, \varphi_{j, \Lambda}\right\rangle_{m, \Lambda} U_{\Lambda} \varphi_{j, \Lambda} .
\end{aligned}
$$

We are now in a position to prove the theorem.

Proof of Theorem 1. Let $\left(w_{t},\left\{P_{x}\right\}_{x \in X}\right)$ be a continuous-time random walk with generator $P-I$ and $\left(\left(w_{t}, A_{t}\right),\left\{Q_{\eta}\right\}_{\eta \in Y}\right)$ a continuous-time random walk with generator $Q-I$. Set $F_{\lambda}(\eta)=E_{\eta}\left[e^{-\lambda T_{N}} ; w_{T_{N}}=\xi\right](\lambda>0)$ for $\eta \in Y$ and for a fixed $\xi \in L_{N}$, where $T_{N}$ is the first hitting time to the set $L_{N}$. Since $F_{\lambda}(\eta)=\delta_{\xi}(\eta)$ for $\eta \in L_{N}$, it is easy to see by the strong Markov property that it satisfies the following linear equation with boundary conditions

$$
(\lambda+1) F_{\lambda}=R F_{\lambda}+S \delta_{\xi}, \quad \text { on } Y \backslash L_{N} .
$$

Since the maximal eigenvalue of $R$ is strictly less than 1 , we obtain

$$
F_{\lambda}=(\lambda+1-R)^{-1} S \delta_{\xi}=\int_{0}^{\infty} e^{-\lambda t} e^{-(I-R) t} S \delta_{\xi} d t
$$

for $\lambda \geq 0$. Therefore,

$$
\frac{Q_{\eta}\left(T_{N} \in d t ; w_{T_{N}}=\xi\right)}{d t}=e^{-(I-R) t} S \delta_{\xi}(\eta) .
$$

In particular, if we take $\eta=(x,\{x\})$ and $\xi=(y, X)$, we obtain

$$
\begin{aligned}
& \frac{P_{x}\left(C_{X} \in d t ; w_{C_{X}}=y\right)}{d t} \\
& =e^{-(I-R) t} S U_{X} \delta_{y}(x,\{x\}) \\
& \quad=\sum_{\Lambda \in \mathcal{D}_{x}}(-1)^{N-|\Lambda|+1} \sum_{j=1}^{|\Lambda|}\left\langle P_{\Lambda} \Lambda^{c} \delta_{y}, \varphi_{j, \Lambda}\right\rangle_{m, \Lambda} e^{-(I-R) t} U_{\Lambda} \varphi_{j, \Lambda}(x,\{x\})
\end{aligned}
$$

by the equality $\Pi_{2} U_{X} \delta_{y}=\delta_{\xi}$ and Lemma 8 . Here $\sum_{\Lambda \in \mathcal{D}}$ is replaced with $\sum_{\Lambda \in \mathcal{D}_{x}}$ since $U_{\Lambda} \varphi_{j, \Lambda}(x,\{x\})=0$ unless $x \in \Lambda$. We note that

$$
e^{-(I-R) t} U_{\Lambda} \varphi_{j, \Lambda}(x,\{x\})=e^{-\left(I-P_{\Lambda}\right) t} \varphi_{j, \Lambda}(x)=\frac{1}{m(x)}\left\langle e^{\Delta_{\Lambda} t} \delta_{x}, \varphi_{j, \Lambda}\right\rangle_{m, \Lambda}
$$

for $\Lambda \in \mathcal{D}_{x}$. Hence, we obtain

$$
\frac{P_{x}\left(C_{X} \in d t ; w_{C_{X}}=y\right)}{d t}=\sum_{\Lambda \in \mathcal{D}_{x}}(-1)^{N-|\Lambda|+1} \frac{1}{m(x)}\left\langle P_{\Lambda \Lambda^{c}} \delta_{y}, e^{\Delta_{\Lambda} t} \delta_{x}\right\rangle_{m, \Lambda} .
$$


We denote by $p_{t}^{\Lambda}(x, y)$ the transition kernel of the random walk in $\Lambda$ with generator $\Delta_{\Lambda}$. Since $P_{\Lambda} \Lambda^{c} \delta_{y}(z)=p(z, y) I_{\Lambda^{c}}(y)$ for $z \in \Lambda$ and $p_{t}^{\Lambda}(x, y)$ is $\left.m\right|_{\Lambda}$-symmetric, we have

$$
\begin{aligned}
\frac{1}{m(x)}\left\langle P_{\Lambda} \Lambda^{c} \delta_{y}, e^{\Delta_{\Lambda} t} \delta_{x}\right\rangle_{m, \Lambda} & =\frac{1}{m(x)} \sum_{z \in \Lambda} p(z, y) I_{\Lambda^{c}}(y) p_{t}^{\Lambda}(z, x) m(z) \\
& =\sum_{z \in \Lambda} p_{t}^{\Lambda}(x, z) p(z, y) I_{\Lambda^{c}}(y) \\
& =\frac{P_{x}\left(T_{\Lambda} \in d t, w_{T_{\Lambda}}=y\right)}{d t}
\end{aligned}
$$

Hence, replacing $\mathcal{D}_{x}$ with $\mathcal{D}_{x} \backslash \mathcal{D}_{y}$ in (3.1), we obtain (1.1).

Proof of Corollary 1. Note that $P_{\Lambda \Lambda^{c}} 1=-\Delta_{\Lambda} \mathbf{1}_{\Lambda}$ since $P 1=1$. By summing up (3.1) over $y \in X$, we see that

$$
\begin{aligned}
\frac{P_{x}\left(C_{X} \in d t\right)}{d t} & =\sum_{\Lambda \in \mathcal{D}_{x}}(-1)^{N-|\Lambda|+1} \frac{1}{m(x)}\left\langle P_{\Lambda \Lambda^{c}} 1, e^{t \Delta_{\Lambda}} \delta_{x}\right\rangle_{m, \Lambda} \\
& =\sum_{\Lambda \in \mathcal{D}_{x}}(-1)^{N-|\Lambda|+1} \frac{1}{m(x)}\left\langle\mathbf{1}_{\Lambda},-\Delta_{\Lambda} e^{t \Delta_{\Lambda}} \delta_{x}\right\rangle_{m, \Lambda} \\
& =\sum_{\Lambda \in \mathcal{D}_{x}}(-1)^{N-|\Lambda|+1} \sum_{i=1}^{|\Lambda|}\left\langle\mathbf{1}_{\Lambda}, \varphi_{i, \Lambda}\right\rangle_{m, \Lambda} \xi_{i, \Lambda} e^{-\xi_{i, \Lambda} t} \varphi_{i, \Lambda}(x) .
\end{aligned}
$$

Acknowledgements. The research of TS was partially supported by JSPS under the Grantin-Aid for Scientific Research No.18540130.

\section{REFERENCES}

[1] D. J. Aldous. An introduction to covering problem for random walks on graphs. J. Theoret. Prob. 2 (1989), 87-89.

[2] D. J. Aldous and J. Fill. Reversible Markov chains and random walks on graphs. http://stat-www.berkeley.edu/users/aldous/RWG/book.html, 2001.

[3] A. Dembo. Favorite Points, Cover Times and Fractals (Lecture Notes in Mathematics, 1869). Springer, Berlin, 2005, pp. 3-101.

[4] W. Feller. The asymptotic distribution of the range of sums of independent random variables. Ann. Math. Statist. 22 (1951), 427-432.

[5] L. Lovász and P. Winkler. A note on the last new vertex visited by a random walk. J. Graph Theory 17 (1993), 593-596.

[6] D. Revuz and M. Yor. Continuous martingales and Brownian motion, 2nd edn. Springer, Berlin, 1994. 
T. Ohwa and T. Shirai

Takuya Ohwa

Faculty of Mathematics

Kyushu University

6-10-1 Hakozaki, Higashi-ku

Fukuoka 812-8581, Japan

(E-mail:ohwa@math.kyushu-u.ac.jp)

Tomoyuki Shirai

Faculty of Mathematics

Kyushu University

6-10-1 Hakozaki, Higashi-ku

Fukuoka 812-8581, Japan

(E-mail: shirai@math.kyushu-u.ac.jp) 